\title{
Civic educating rural pregnant mothers to take full nevaripine dose a tool to reducing HIV prevalence in Malawi
}

\author{
Caleb Kondwani Faith Thole 1,2,3 \\ From $16^{\text {th }}$ International Symposium on HIV and Emerging Infectious Diseases \\ Marseille, France. 24-26 March 2010
}

\section{Background}

It is estimated that 600,000 women get pregnant every year in Malawi. Primary health care plays a vital role. Prevention to Child Transmission of HIV education is important in a country like Malawi where prevalence of HIV positive mothers exceeds $20 \%$ than normal country prevalence of $12 \%$. In the ultra-partum period Nevaripine has proved to be an effective drug in reducing transmission from mother to Child. Accessibility of essential medicine like nevaripine drug to women and neonates is especially a problem to rural areas where many women deliver at home due to difficulties in accessing health facilities.

\section{Objectives}

This civic education intervention aimed to increase rural HIV+ pregnant mothers uptake of Nevarapine tablets and syrup for infants' dose for Prevention of mother to child transmission of HIV and to promote hospital delivery.

\section{Methods}

Global Hope Mobilization is providing education and counselling to women in rural setting to access basic medicines such as nevaripine, as well as to promote hospital delivery in Lilongwe district rural setting. Households with pregnant women were included through a random sample and a total of 700 pregnant women were included in a programme to provide civic education materials, including leaflets; local meetings, counselling sessions, and home visits. Nevaripine tablets and syrup were also administered, ANC data were collected and used from the nearest health facility. The intervention was assessed through one to one interviews, focus group discussion, and questionnaires.

\section{Results}

$100 \%$ of the pregnant women were sensitized on taking nevaripine, counselled and referred for HIV testing. To date $98 \%$ of women have attended pre and post VCT counselling testing and ANC at health facilities and at private clinics referred to. Of these women $66 \%$ were found to be HIV positive. Nevaripine tablets and instructions were issued to them when they reached 32 to 34 weeks of gestation. $60 \%$ Infants received Nevaripine syrup within 3 days after birth. Of these $6 \%$ are not known to have taken syrup and were lost to follow up, while $60 \%$ returned for post counselling. The major barriers of the intervention were home delivery, traditional beliefs and lack of knowledge of HIV status and on the course of nevaripine.

\section{Discussion}

The main risk factor for lack of full take up of Nevaripine for HIV+ women is home delivery and lack of knowledge on their HIV status. A civic education on Nevaripine and hospital delivery among HIV positive pregnant women in this rural population setting can reduce HIV transmission from mother to child.

\section{Author details}

${ }^{1}$ Global Hope Mobilization, Lilongwe, Malawi. ${ }^{2}$ International AIDS Society, Geneva, Switzerland. ${ }^{3}$ Equinet, Soutthern Africa, Zimbabwe.

Published: 11 May 2010

doi:10.1186/1742-4690-7-S1-P140

Cite this article as: Thole: Civic educating rural pregnant mothers to

take full nevaripine dose a tool to reducing HIV prevalence in Malawi. Retrovirology 2010 7(Suppl 1):P140. 